\title{
TU/e emonownen

\section{Analysis of the vibronic fine structure in circularly polarized emission spectra from chiral molecular aggregates}

\section{Citation for published version (APA):}

Spano, F. C., Zhao, Z., \& Meskers, S. C. J. (2004). Analysis of the vibronic fine structure in circularly polarized emission spectra from chiral molecular aggregates. Journal of Chemical Physics, 120(22), 10594-10604. https://doi.org/10.1063/1.1730114

DOI:

10.1063/1.1730114

Document status and date:

Published: 01/01/2004

\section{Document Version:}

Publisher's PDF, also known as Version of Record (includes final page, issue and volume numbers)

\section{Please check the document version of this publication:}

- A submitted manuscript is the version of the article upon submission and before peer-review. There can be important differences between the submitted version and the official published version of record. People interested in the research are advised to contact the author for the final version of the publication, or visit the $\mathrm{DOI}$ to the publisher's website.

- The final author version and the galley proof are versions of the publication after peer review.

- The final published version features the final layout of the paper including the volume, issue and page numbers.

Link to publication

\section{General rights}

Copyright and moral rights for the publications made accessible in the public portal are retained by the authors and/or other copyright owners and it is a condition of accessing publications that users recognise and abide by the legal requirements associated with these rights.

- Users may download and print one copy of any publication from the public portal for the purpose of private study or research.

- You may not further distribute the material or use it for any profit-making activity or commercial gain

- You may freely distribute the URL identifying the publication in the public portal.

If the publication is distributed under the terms of Article $25 \mathrm{fa}$ of the Dutch Copyright Act, indicated by the "Taverne" license above, please follow below link for the End User Agreement:

www.tue.nl/taverne

Take down policy

If you believe that this document breaches copyright please contact us at:

openaccess@tue.nl

providing details and we will investigate your claim. 


\title{
Analysis of the vibronic fine structure in circularly polarized emission spectra from chiral molecular aggregates
}

\author{
Frank C. Spano a) and Zhen Zhao \\ Department of Chemistry, Temple University, Philadelphia, Pennsylvania 19122 \\ Stefan C. J. Meskers ${ }^{\text {b) }}$ \\ Laboratory for Macromolecular an Organic Chemistry, Eindhoven University of Technology, \\ The Netherlands
}

(Received 22 January 2004; accepted 9 March 2004)

\begin{abstract}
Using a Frenkel-exciton model, the degree of circular polarization of the luminescence $\left(g_{\text {lum }}\right)$ from one-dimensional, helical aggregates of chromophoric molecules is investigated theoretically. The coupling between the electronic excitation and a local, intramolecular vibrational mode is taken into account. Analytical expressions for the fluorescence band shape and $g_{\text {lum }}$ are presented for the case of strong and weak electronic coupling between the chromophoric units. Results are compared to those from numerical calculations obtained using the three particle approximation. $g_{\text {lum }}$ for the $0-0$ vibronic band is found to be independent of the relative strength of electronic coupling between chromophores and excitation-vibration coupling. It depends solely on the number of coherently coupled molecules. In contrast, for the higher vibronic transitions $\left|g_{\text {lum }}\right|$ decreases with decreasing strength of the electronic coupling. In the limit of strong electronic coupling, $\left|g_{\text {lum }}\right|$ is almost constant throughout the series of vibronic transitions but for weak coupling $\left|g_{\text {lum }}\right|$ becomes vanishingly small for all vibronic transitions except for the 0-0 transition. The results are interpreted in terms of dynamic localization of the excitation during the zero point vibrational motion in the excited state of the aggregate. It is concluded that circular polarization measurements provide an independent way to determine the coherence size and bandwidth of the lowest exciton state for chiral aggregates. (C) 2004 American Institute of Physics. [DOI: 10.1063/1.1730114]
\end{abstract}

\section{INTRODUCTION}

In view of their industrial applications in, e.g., display devices, the optical properties of $\pi$-conjugated, semiconducting polymers have been studied intensively in the last decade. ${ }^{1-3}$ A considerable number of studies have been devoted to understanding the effect of electronic interactions between chains of conjugated polymer on the luminescence quantum yield of solid films of these polymers. ${ }^{4}$ There seems to be consensus now that interchain interactions can lead to excitations that are delocalized over more than one polymer chain. The luminescence efficiency of these interchain species can in certain cases be considerably lower than for an isolated chain. Therefore, understanding of interchain electronic coupling is of considerable practical importance.

Chiral $\pi$-conjugated polymers constitute a special class of macromolecules. ${ }^{5}$ Due to the absence of mirror image symmetry at the local structural level, a randomly oriented ensemble of these polymers shows a difference in absorption of left and right circular polarized light. In the case of fluorescent polymers, left and right polarized light are also emitted with a different probability. Structural characteristics of these chiral polymers can be probed by chiroptical techniques, such as circular dichroism (CD) and circularly polarized luminescence (CPL) which are sensitive to chirality in the ground and excited state, respectively. 6,7 Using these techniques, a large number of chiral polymers have been

\footnotetext{
a)Electronic mail: spano@temple.edu

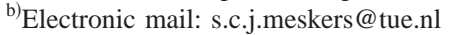

investigated. For many polymers, CD and CPL are practically zero for molecularly dissolved chains. However, upon formation of nano-aggregates in poor solvent, significant CD and CPL effects can be observed. ${ }^{8}$ It may therefore be expected that circular polarization effects are very sensitive to interchain interactions. Due to the disordered nature of the polymer chains, quantitative analysis of the chiroptical data is very difficult.

Apart from polymers, also chiral $\pi$-conjugated oligomers with a well-defined structure have been investigated using chiroptical spectroscopy. ${ }^{9-13}$ These molecules have a tendency to form aggregates and related superstructures. There is growing evidence that also cyanine dye molecules can be organized into chiral structures resulting in chiroptical properties. ${ }^{14,15}$ The circular polarization differentials contain, in principle, information on the structure of the aggregate.

For small chiral molecules containing a single chromophoric group, the electronic circular dichroism can be calculated from first principles using modern quantum chemical methods ${ }^{16,17}$ and information can be extracted from the rather subtle CD effects. Aggregates of many chromophoric molecules are systems that are in most cases too large to be amenable to quantum chemical calculations using, e.g., molecular orbital or density functional methods. Instead, circular polarization effects are usually modeled in terms of exciton theory. ${ }^{18}$ For aggregates, the relation between structure of the aggregate and chiroptical spectra has been analyzed $^{19,20}$ and, especially, molecules/aggregates containing two identical chromophoric groups have been investi- 
gated extensively ${ }^{21}$ with the aim of determining the absolute stereochemistry of the molecule from chiroptical data. Briefly, when two identical chromophores without stereocenters are held together by a chiral scaffold, delocalization of the excitation over the two chromophoric groups induces a circular differential polarization in absorption and emission. Thus, the degree of circular polarization is a sensitive measure of the delocalization of the photoexcitation over the chromophoric groups in the cluster.

The coupling between electronic and nuclear motion in such aggregates is a serious problem in the description of the excited state of such multichromophoric arrays. ${ }^{22-24}$ As a result of this coupling, the Born-Oppenheimer approximation becomes inaccurate and in many cases one has to resort to numerical methods to model absorption and luminescence spectra. ${ }^{25-28}$ For $\pi$-conjugated oligomers, coupling between electronic excitations and vibrations is usually quite strong, resulting in the appearance of vibronic progressions in the optical spectra of these materials. For aggregates, this fine structure contains, in principle, information on the degree of delocalization and the dynamical behavior of a photo excitation on the aggregate. Its study is therefore of considerable importance.

Here we focus on the effect of vibronic coupling on the chiroptical properties of chiral aggregates of chromophoric molecules. Some studies on vibronic effects in CD have appeared, ${ }^{29-32}$ yet no detailed analysis of vibronic effects in CPL has been given. As fluorescence usually occurs from the lowest electronically excited state, CPL spectra are often considerably easier to interpret than $\mathrm{CD}$ spectra since they involve only a single electronic transition. Here, we will show that vibronic coupling can lead to a pronounced wavelength dependence of the degree of circular polarization in the fluorescence band from a chiral aggregate. These effects can be interpreted in terms of dynamic localization where the degree of delocalization of an excitation is strongly coupled to the zero-point vibrational motion in the excited state. The calculations indicate that polaronic effects can play an important role in the dynamics of the excitations.

\section{MODEL AGGREGATES}

In this study we consider linear aggregates composed of achiral rod-shaped conjugated molecules such as the unsubstituted oligo-phenylene vinylenes or oligothiophenes, where the molecular plane of each molecule is oriented normal to the aggregate axis $z$. Molecules are labeled with the index, $n$, with $n=1,2, \ldots, N$, where $N$ is the total number of molecules within the aggregate. The nearest neighbor separation is $d$ and the aggregate length, $(N-1) d$, is taken to be much smaller than an optical wavelength. Chirality results from the helical nature of the aggregate created by rotating molecule $n$ 's long axis by an amount proportional to $n$, as demonstrated in Fig. 1. The orientation of molecule $n$ 's long axis is given by $\phi n$, where $\phi$ is the pitch angle. If we further assume centrosymmetric molecules, then $\phi$ and $\phi+\pi$ are indistinguishable, making $\phi$ range from $-\pi / 2$ to $\pi / 2$.

In what follows each molecule in the aggregate is taken to be a two-level system consisting of a ground electronic state, $1{ }^{1} A_{g}$, and an excited electronic state, $1{ }^{1} B_{u}$. The (op-

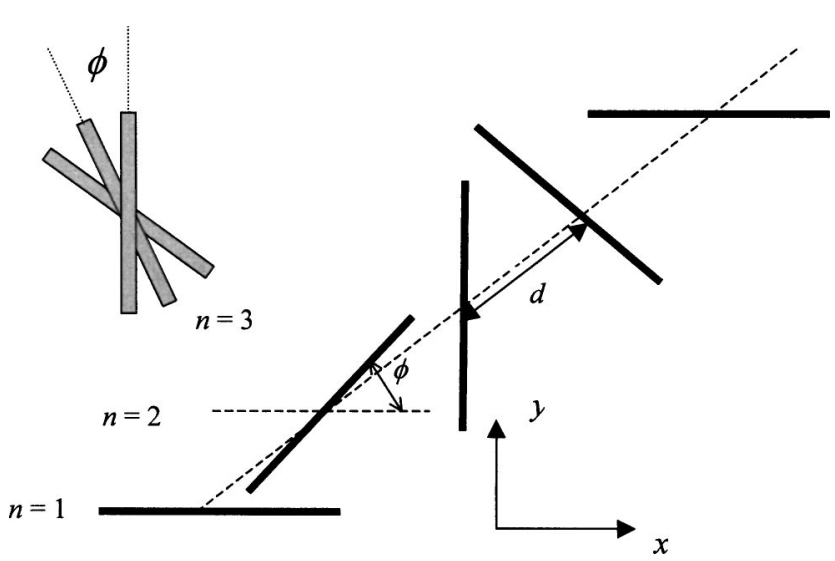

FIG. 1. Model aggregate considered in this work with lines representing long molecular axis.

tical gap) transition between the two is coupled to a single totally symmetric intramolecular vibration with frequency, $\omega_{0}$. The coupling is caused by a shift in the nuclear potential well, taken to be harmonic, upon electronic excitation and is quantified by the Huang-Rhys factor, $\lambda^{2}$. The ground and excited harmonic wells are assumed to be of the same curvature (i.e., no change in $\omega_{0}$ with electronic excitation).

Within the aggregate an excited molecule at site $m$ can resonantly transfer its energy to molecule at $n$ via the excitonic coupling, $J_{m n}$. The aggregate Hamiltonian, written in a representation of one-excitons, reads

$$
\begin{aligned}
H= & \omega_{0} \sum_{n} b_{n}^{\dagger} b_{n}+\omega_{0} \lambda \sum_{n}\left(b_{n}^{\dagger}+b_{n}\right)|n\rangle\langle n| \\
& +\sum_{m} \sum_{n} J_{m n}|m\rangle\langle n|+D+\omega_{0-0}+\lambda^{2} \omega_{0},
\end{aligned}
$$

where $\hbar=1$ is taken. $b_{n}^{\dagger}\left(b_{n}\right)$ is the creation (destruction) operator for the harmonic oscillator representing the ground state nuclear potential on molecule $n$. The electronic state, $|n\rangle$, with $|n\rangle \equiv\left|e_{n}\right\rangle \Pi_{m \neq n}\left|g_{m}\right\rangle$, indicates that the molecule at $n$ is electronically excited to the state $1{ }^{1} B_{u}$ (alias $e_{n}$ ), while all other molecules remain in their electronic ground states, $1{ }^{1} A_{g}$ (alias states $\left.g_{m}\right) . \omega_{0-0}$ is the gas-phase, $1^{1} A_{g} \rightarrow 1^{1} B_{u}$, 0-0 transition frequency, and $D$ is the gas-to-crystal (red) shift of the transition energy due to nonresonant secondorder dispersion forces, assumed the same for all molecules in the aggregate.

In what follows we further assume that exciton coupling is limited to nearest neighbors,

$$
J_{0} \equiv J_{n, n \pm 1}=J_{n \pm 1, n}
$$

and that $J_{0}>0$. If periodic boundary conditions are invoked, then we also have $J_{1, N}=J_{N, 1}=J_{0}$. Maintaining positive $J_{0}$ places the $k=\pi$ exciton at the bottom of the band, as in $\mathrm{H}$-aggregates. The free exciton bandwidth $W$ is given by $W$ $=4 J_{0}$ for $N>2$ and by $W=2 J_{0}$ for dimers $(N=2)$.

\section{AGGREGATE EMISSION}

To analyze the Hamiltonian (1) we use the multi-particle basis $\operatorname{set}^{33-35}$ based on the total number of vibronically and vibrationally excited molecules. One-particle states, denoted, 


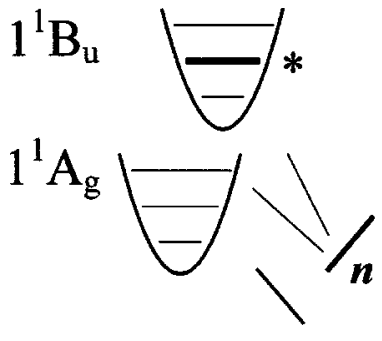

\section{One-particle states}

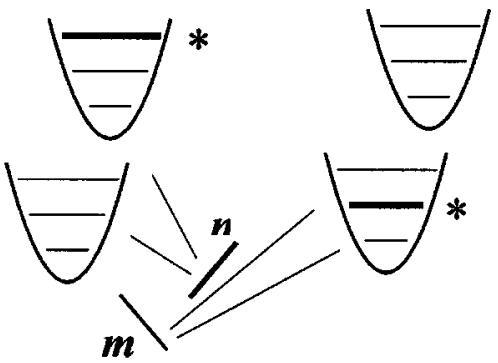

Two-particle states

FIG. 2. Schematic representation of one-and two-particle states, $|n, \widetilde{v}=1\rangle$ and $|n, \widetilde{v}=2 ; m, v=1\rangle$, respectively.

$|n, \widetilde{v}\rangle$, consist of a single vibronic excitation at site $n$ (with all other sites electronically and vibrationally unexcited). The vibronic excitation involves $\widetilde{v}(\geqslant 0)$ vibrational quanta in the excited $\left(1^{1} B_{u}\right)$ nuclear potential, which for a totally symmetric vibration is generally shifted from the ground state $\left(1^{1} A_{g}\right)$ nuclear potential. Two-particle states, denoted $\left|n, \widetilde{v} ; n^{\prime}, v^{\prime}\right\rangle$, consist of a vibronic excitation at site $n$ and a vibrational excitation at site $n^{\prime}$ (with $n^{\prime} \neq n$ ). The latter involves $v^{\prime}(\geqslant 1)$ vibrational quanta in the ground state nuclear well. Continuing in this fashion, the $p$ th particle state consists of a single vibronic excitation and $p-1$ vibrational excitations with all $p$ excitations (vibronic and vibrational) occurring on distinct molecules. Figure 2 gives a schematic representation of one- and two-particle states.

When $\left|J_{0}\right|<\omega_{0}$ truncating the multi-particle basis set to include only one- and two-particle states, the so-called twoparticle approximation, gives accurate absorption and emission spectra. ${ }^{34}$ Larger excitonic couplings require three and higher particle states, primarily for attaining accurate spectral properties of the low (high) energy excitons in aggregates with $J_{0}>0\left(J_{0}<0\right)$. For this work we investigate aggregates with $J_{0}$ as large as $10 \omega_{0}$, requiring up to three-particle excitations. Consequently, the wave function of the $\alpha$ th vibrationally dressed exciton is written as

$$
\begin{aligned}
\left|\Psi^{(\alpha)}\right\rangle= & \sum_{n} \sum_{\widetilde{v}} c_{n \tilde{v}}^{(\alpha)}|n, \widetilde{v}\rangle+\sum_{n, \tilde{v}} \sum_{n^{\prime}, v^{\prime}} c_{n \tilde{v} ; n^{\prime}, v^{\prime}}^{(\alpha)}\left|n, \widetilde{v} ; n^{\prime}, v^{\prime}\right\rangle \\
& +\sum_{n, \widetilde{v}} \sum_{n^{\prime}, v^{\prime}} \sum_{n^{\prime \prime}, v^{\prime \prime}} c_{n \tilde{v} ; n^{\prime}, v^{\prime} ; n^{\prime \prime}, v^{\prime \prime}}^{(\alpha)}\left|n, \widetilde{v} ; n^{\prime}, v^{\prime} ; n^{\prime \prime}, v^{\prime \prime}\right\rangle
\end{aligned}
$$

with energy, $E^{(\alpha)}$. In the second (two-particle) term and third (three-particle) term the sums over molecular sites are restricted so that $n^{\prime} \neq n$ in the second term, and $n, n^{\prime}, n^{\prime \prime}$ are all different in the third term. In addition, the vibrational excitations must contain at least one quanta for the two- and three-particle excitations. Thus, $v^{\prime} \geqslant 1$ in the second term and $v^{\prime}, v^{\prime \prime} \geqslant 1$ in the third.

In this work we treat defect-free aggregates at low temperature. In this case, emission emanates from the lowest energy singlet exciton, denoted $\left|\Psi^{(e m)}\right\rangle$, with transition energy from the vibrationless ground state, $\omega_{e m}$. For $J_{0}>0$, the emitting state contains the phase alternation characteristic of a wave vector $k=\pi$. [For example, $c_{n \tilde{v}}^{(e m)}=(-1)^{n}\left|c_{n \tilde{v}}^{(e m)}\right|$.] In what follows we investigate the frequency-dependent total luminescence, $S(\omega)$, as well as the dissymmetry factor for circularly polarized luminescence, $g_{\text {lum }}$.

In dimensionless units the unpolarized steady-state emission spectrum is determined from

$$
\begin{aligned}
S(\omega)= & \sum_{v_{t}=0,1,2, \ldots} I^{0-v_{t}}\left(1-v_{t} \omega_{0} / \omega_{e m}\right)^{3} W_{e}\left(\omega-\omega_{e m}+v_{t} \omega_{0}\right) / \\
& W_{e}(0) .
\end{aligned}
$$

The sum is over all $0-v_{t}$ transitions, where $v_{t}$ is the number of ground state vibrational quanta in the terminal state of the emission process. $W_{e}(\omega)$ is a symmetric line shape function and $I^{0-v_{t}}$ is the dimensionless line (or oscillator) strength for the $0-v_{t}$ transition, given by

$$
I^{0-v_{t}}=\frac{1}{\mu_{0}^{2}} \sum_{\left\{v_{n}\right\}}^{\prime} \mid\left\langle\Psi^{(e m)}\left|\hat{\mathbf{M}} \prod_{n}\right| g_{n}, v_{n} \mid\right\rangle^{2} .
$$

$\Pi_{n}\left|g_{n}, v_{n}\right\rangle$ represents a terminal state in the emission event. The sum in Eq. (4) is over all terminal states, each identified by a unique set of quantum numbers $\left\{v_{n}\right\}=\left\{v_{1}, v_{2}, \ldots, v_{N}\right\}$, under the constraint $\Sigma_{n} v_{n}=v_{t}$ indicated by the prime. Finally, $\hat{M}$ is the aggregate transition dipole moment (tdm) operator,

$$
\hat{\mathbf{M}}=\sum_{n} \hat{\boldsymbol{\mu}}_{n},
$$

with the site tdm operator defined as

$$
\hat{\boldsymbol{\mu}}_{n} \equiv\left(\mu_{n, x} \mathbf{i}+\mu_{n, y} \mathbf{j}\right)|n\rangle\langle g|+\text { h.c. },
$$

Assuming the molecular tdm lies along the long molecular axis, the components of the tdm of the $n$th molecule are

$$
\mu_{n, x}=\mu_{0} \cos (\phi n),
$$

and

$$
\mu_{n, y}=\mu_{0} \sin (\phi n),
$$

where $\phi$ is defined in Fig. 1.

In addition to the emission intensity, we are also interested in the degree of circular polarization in the $0-v_{t}$ luminescence peak, denoted by the dissymmetry factor, $g_{\text {lum }}^{0-v_{t}}$. In the general case, $g_{\text {lum }}^{0-v_{t}}$ depends on the polarization of the exciting light, the geometry of the excitation-detection scheme, and the orientational distribution of chromophores. 
In the simplest case of an isotropic distribution of aggregates undergoing rapid rotational diffusion (so that photoselection can be ignored), $g_{\text {lum }}^{0-v_{t}}$ is given by ${ }^{36,37}$

$$
g_{\text {lum }}^{0-v_{t}}=2\left(W_{L}^{0-v_{t}}-W_{R}^{0-v_{t}}\right) /\left(W_{L}^{0-v_{t}}+W_{R}^{0-v_{t}}\right),
$$

where $W_{L}^{0-v_{t}}\left(W_{R}^{0-v_{t} t}\right)$ is the peak intensity of left-handed (right-handed) circularly polarized emission corresponding to the $0-v_{t}$ transition. $g_{\text {lum }}^{0-v_{t}}$ in Eq. (7) can be evaluated by generalizing the dimer formalism presented by Craig and Thirunamachandran ${ }^{38}$ to an aggregate of $N$ molecules. The result is

$$
g_{\text {lum }}^{0-v_{t}}=\frac{2 \sum_{\left\{v_{m}\right\}}^{\prime} \sum_{n, n^{\prime}} K_{n-n^{\prime}} \boldsymbol{\mu}_{n^{\prime}}^{\left\{v_{m}\right\}, e m} \times \boldsymbol{\mu}_{n}^{e m,\left\{v_{m}\right\}} \cdot \mathbf{r}_{n n^{\prime}}}{\sum_{\left\{v_{m}\right\}}^{\prime} \sum_{n, n^{\prime}} G_{n-n^{\prime}} \boldsymbol{\mu}_{n}^{\left\{v_{m}\right\}, e m} \cdot \boldsymbol{\mu}_{n^{\prime}}^{e m,\left\{v_{m}\right\}}},
$$

where $\mathbf{r}_{n n^{\prime}}$ is the unit vector, $\mathbf{r}_{n n^{\prime}} \equiv\left(\mathbf{r}_{n}-\mathbf{r}_{n^{\prime}}\right) /\left|\mathbf{r}_{n}-\mathbf{r}_{n^{\prime}}\right|$, connecting sites $n$ and $n^{\prime}$, and $\boldsymbol{\mu}_{n}^{\left\{v_{m}\right\}, e m}$ is the matrix element of $\hat{\boldsymbol{\mu}}_{n}$ in Eq. (5b) between the emitting state and the ground electronic state containing the set $\left\{v_{m}\right\}$ of vibrational excitations,

$$
\boldsymbol{\mu}_{n}^{\left\{v_{m}\right\}, e m} \equiv \prod_{m}\left\langle g_{m}, v_{m}\left|\hat{\boldsymbol{\mu}}_{n}\right| \Psi^{(e m)}\right\rangle .
$$

Unlike the line strengths in Eq. (4), the dissymmetry in Eq. (8) does not assume the aggregate length to be much smaller than the emission wavelength. The generalization is necessary in order to treat special aggregate sizes in which the $0-v_{t}$ emission intensity becomes zero, as will be explained below. The factors $G_{n}$ and $K_{n}$ in Eq. (8) take into account extended size effects, important when the size of the aggregate compares with the optical wavelength $2 \pi / k$ with $k$ $\equiv \omega_{0-0} / c$ :

$$
G_{n}=\frac{\sin (k d n)}{k d n}+\frac{\cos (k d n)}{(k d n)^{2}}-\frac{\sin (k d n)}{(k d n)^{3}}
$$

and

$$
K_{n}=-\frac{\cos (k d n)}{k d n}+\frac{\sin (k d n)}{(k d n)^{2}},
$$

with $d$ is the nearest neighbor distance (see Fig. 1). In the small aggregate limit $[k d(N-1) \ll 1], G_{n}$ and $K_{n}$ approach $\frac{2}{3}$ and $k d n / 3$, respectively. In the same limit the denominator in Eq. (8) approaches $2 \mu_{0}^{2} I^{0-v_{t} / 3}$ and the dissymmetry reduces to the more familiar form,

$$
\begin{aligned}
g_{\text {lum }}^{0-v_{t}}= & \frac{4 i}{c \mu_{0}^{2} I^{0-v_{t}}} \sum_{\left\{v_{n}\right\}}{ }^{\prime}\left\langle\Psi^{(e m)}\left|\hat{\mathbf{M}} \prod_{n}\right| g_{n} ; v_{n}\right\rangle \\
& \cdot \prod_{n}\left\langle g_{n} ; v_{n}|\hat{\mathbf{m}}| \Psi^{(e m)}\right\rangle .
\end{aligned}
$$

The sum in Eq. (11) is the rotational strength of the $0-v_{t}$ transition, depending on both the electric and magnetic dipole moments. When the transition of the individual chromophores can be treated in the electric dipole approximation and when electron exchange between the two chromophores is neglected, the magnetic dipole moment operator $\hat{\mathbf{m}}$ is ${ }^{38}$

$$
\hat{\mathbf{m}}=\frac{i c k}{2} \sum_{n}|n\rangle\langle g| \mathbf{r}_{n} \times \boldsymbol{\mu}_{n}+\text { h.c. }
$$

with $\boldsymbol{\mu}_{n} \equiv \mu_{n, x} \mathbf{i}+\mu_{n, y} \mathbf{j}$. The small aggregate expression for $g_{\text {lum }}^{0-v_{t}}$ in Eq. (11) is applicable whenever $I^{0-v_{t}}$ is not close to zero. When $I^{0-v_{t}}=0$ the more general, extended aggregate form of $g_{\text {lum }}^{0-v_{t}}$ in Eq. (8) must be employed in order to avoid the unphysical divergence predicted by Eq. (11), as described below.

\section{PROPERTIES OF THE 0-0 AND 0-1 EMISSION}

In this Section we analyze in greater detail the more accessible $0-0$ and $0-1$ emission line strengths and dissymmetry factors. We begin by presenting general expressions for $I^{0-0}, I^{0-1}, g_{\text {lum }}^{0-0}$ and $g_{\text {lum }}^{0-1}$ and then obtain simpler analytical expressions by invoking periodic boundary conditions (pbc's). Unless explicitly stated we assume the small aggregate limit with $k d(N-1) \ll 1$.

Inserting $\left|\Psi^{(e m)}\right\rangle$ from Eq. (2) into Eqs. (4) and (11) with $v_{t}=0$ gives the general expressions for the 0-0 line strength and luminescence dissymmetry:

$$
\begin{aligned}
I^{0-0}= & \mu_{0}^{-2} \sum_{\beta}\left|\sum_{n, \tilde{v}} \mu_{n, \beta} f_{0 \tilde{v}} c_{n, \tilde{v}}^{(e m)}\right|^{2} \\
g_{\text {lum }}^{0-0}= & \frac{2 k}{\mu_{0}^{2} I^{0-0}} \sum_{n>n^{\prime}} \sum_{\tilde{v}, \tilde{v}^{\prime}} f_{0 \tilde{v}} f_{\tilde{v}^{\prime} 0} c_{n, \tilde{v}}^{(e m)} c_{n^{\prime}, \tilde{v}^{\prime}}^{(e m)}\left(\boldsymbol{\mu}_{n^{\prime}} \times \boldsymbol{\mu}_{n}\right) \\
& \cdot\left(\mathbf{r}_{\mathbf{n}}-\mathbf{r}_{\mathbf{n}^{\prime}}\right),
\end{aligned}
$$

where we assume, without loss in generality, real wave function coefficients. In Eqs. (13a) and (13b) we have introduced the vibrational overlap integral, $f_{v^{\prime}} \equiv\left\langle v^{\prime} \mid \widetilde{v}\right\rangle$, between the unshifted $\left(1^{1} A_{g}\right)$ and shifted $\left(1^{1} B_{u}\right)$ harmonic oscillator eigenfunctions with $v^{\prime}$ and $\widetilde{v}$ quanta, respectively. Proceeding with the 0-1 emission we obtain

$$
\begin{aligned}
I^{0-1}= & \mu_{0}^{-2} \sum_{\beta} \sum_{n_{1}} \mid \sum_{\widetilde{v}} \mu_{n_{1}, \beta} f_{1 \tilde{v}} c_{n_{1}, \tilde{v}}^{(e m)} \\
& +\left.\sum_{n_{2}, \tilde{v}} \mu_{n_{2}, \beta} f_{0 \tilde{v}} c_{n_{2} \tilde{v} ; n_{1}, 1}^{(e m)}\right|^{2},
\end{aligned}
$$

for the $0-1$ line strength, and

$$
\begin{aligned}
g_{\text {lum }}^{0-0}= & \frac{2 k}{\mu_{0}^{2} I^{0-1}}\left\{\sum_{n, n^{\prime}} \sum_{\tilde{v}, \tilde{v}^{\prime}} f_{\widetilde{v} 1} f_{0 \tilde{v}^{\prime}} c_{n, \tilde{v})}^{(e m)} c_{n^{\prime}, \tilde{v}^{\prime}, n, 1}^{(e m)}\left(\boldsymbol{\mu}_{n} \times \boldsymbol{\mu}_{n^{\prime}}\right)\right. \\
& \cdot\left(\mathbf{r}_{\mathbf{n}^{\prime}}-\mathbf{r}_{\mathbf{n}}\right) \\
& +\sum_{n} \sum_{n_{1}>n_{2}} \sum_{\tilde{v}_{1}, \widetilde{v}_{2}} f_{\tilde{v}_{1} 0} f_{\tilde{v}_{2} 0} c_{n_{1}, \tilde{v}_{1}, n, 1}^{(e m)} c_{n_{2}, \tilde{v}_{2}, n, 1}^{(e m)} \\
& \left.\times\left(\boldsymbol{\mu}_{n_{1}} \times \boldsymbol{\mu}_{n_{2}}\right) \cdot\left(\mathbf{r}_{n_{2}}-\mathbf{r}_{n_{1}}\right)\right\}
\end{aligned}
$$

for the corresponding dissymmetry. The presence of the vector products in Eqs. (13b) and (14b) shows that the dissymmetry vanishes when the pitch angle $\phi$ is zero, as it should since the aggregate is then no longer chiral. Moreover, Eq. (14b) shows that $g_{\text {lum }}^{0-1}$ lacks a term in the summation due 
solely to single particle coefficients. After substituting the wave function in Eq. (2) into Eq. (11) the resulting expression shows that the pure single-particle contribution is a sum over local dissymmetries involving the vector (dot) product between the electric and magnetic transition dipole moments corresponding to the $\left|e_{n}, \widetilde{v}_{n}\right\rangle \rightarrow\left|g_{n}, v_{n}=1\right\rangle$ transition. Since this transition is confined to the same molecular site the electric and magnetic transition dipoles are normal to each other, making the pure single particle contribution to $g_{\text {lum }}^{0-1}$ vanish.

Equations (13a)-(14b) are also devoid of any threeparticle coefficients. In general, the intensity and dissymmetry of the 0-p emission replica depends only on the $(p+1)$-particle coefficients and lower. However, any given wave function coefficient depends in general on all multiparticle coefficients since in the Hamiltonian $p$-particle states are coupled to the $p-1, p$ and $p+1$ particle states, i.e., a hierarchy of equations which becomes an approximate description when truncated.

Further insight into Eqs. (13) and (14) can be gained from the simpler expressions resulting from the application of pbc's, which we assume from here on. Such boundary conditions impose translational symmetry on the wave functions (despite the lack of translational symmetry in the aggregate), making the exciton wave vector $k$ a good quantum number. If we further take the number of molecules comprising the aggregate, $N$, to be even, the lowest energy (emitting) exciton is nondegenerate with $k=\pi$ when $J_{0}>0$. The single particle coefficients can then be written as

$$
c_{n, \tilde{v}}^{(e m)}=(-1)^{n} c_{\tilde{v}}^{(e m)} / \sqrt{N} .
$$

Using (15) in Eq. (13a) leads to a 0-0 line strength separable into a product of vibrational and electronic parts,

$$
I^{0-0}=\frac{F}{N \mu_{0}^{2}} \sum_{\beta}\left|\sum_{n} \mu_{n, \beta}(-1)^{n}\right|^{2},
$$

where $F$ is a generalized Franck-Condon (FC) factor,

$$
F=\left|\sum_{\widetilde{v}} f_{0 \tilde{v}} c_{\tilde{v}}^{(e m)}\right|^{2},
$$

for 0-0 emission. Evaluating the sum in Eq. (16) using the site tdm's in Eqs. (6a) and (6b) gives the total 0-0 line strength,

$$
I^{0-0}=(F / N) \frac{\sin ^{2}(N \phi / 2)}{\cos ^{2}(\phi / 2)} .
$$

The vanishing of the $0-0$ emission intensity as $\phi$ approaches zero is consistent with the formation of an $\mathrm{H}$-aggregate with a single molecule per unit cell. (Note that in this limit the replicas need not vanish.) For aggregates with $N \phi \ll 1$, the total 0-0 line strength increases linearly with $N$, with $I^{0-0}$ $\rightarrow(F / 4) N \phi^{2}$. Further increases in $N$ result in a damped oscillation with peaks occurring at values of $N$ satisfying $\tan (N \phi / 2)=N \phi$, the first and largest occurring when $N$ $\approx$ int $[2.33 / \phi]$. Minima occur for

$$
N=\operatorname{int}[2 \pi l / \phi], \quad l=1,2, \ldots,
$$

which is the condition for the aggregate to contain (nearly) $l$ full turns. For these values of $N$ the molecular transition
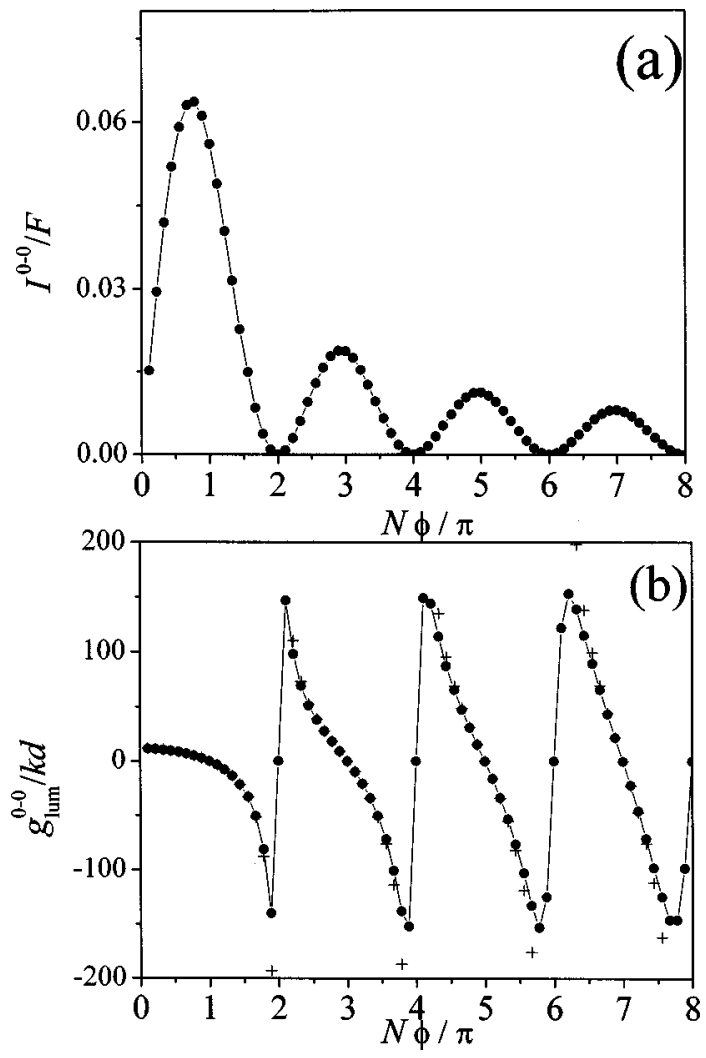

FIG. 3. The normalized $0-0$ intensity line strength, $I^{0-0} / F$ (a), and dissymmetry factor, $g_{l u m}^{0-0} / k d(\mathrm{~b})$, as a function of $N \phi$ using $\phi=\pi / 18 . I^{0-0}$ was evaluated using Eq. (18), while $g_{\text {lum }}^{0-0}$ was calculated using Eq. (20) (crosses) and Eq. (21) (solid circles). In all cases the plots are independent of the strength of the excitonic coupling, $J_{0}$, as well as the strength of the excitonphonon coupling, $\lambda^{2}$ (see text).

dipoles undergo destructive interference. The interference is totally destructive if $2 \pi l / \phi$ is exactly $N$. Figure 3(a) shows the $0-0$ intensity from Eq. (18) as a function of $N$ for $\phi$ $=\pi / 18$.

Turning now to $g_{\text {lum }}^{0-0}$, using Eq. (13b) with (15) and (18) and performing the sum gives a very simple expression for the 0-0 dissymmetry:

$$
g_{\text {lum }}^{0-0}=k d[N \cot (N \phi / 2)+\tan (\phi / 2)] .
$$

Equation (20) derives from the more general expression for an extended aggregate,

$$
g_{\text {lum }}^{0-0}=\frac{2 \sum_{n, n^{\prime}} K_{n-n^{\prime}} \boldsymbol{\mu}_{n^{\prime}} \times \boldsymbol{\mu}_{n} \cdot \mathbf{r}_{n n^{\prime}}}{\sum_{n, n^{\prime}} G_{n-n^{\prime}} \boldsymbol{\mu}_{n} \cdot \boldsymbol{\mu}_{n^{\prime}}}
$$

obtained from Eq. (8). Equation (21) reduces to Eq. (20) in the limit that $k d(N-1) \ll 1$.

$g_{\text {lum }}^{0-0}$ in Eq. (20) or (21) also applies to a free exciton, or an exciton uncoupled from nuclear vibrations $(\lambda=0)$. Hence, the 0-0 dissymmetry (but not the replica dissymmetries, see below) for a dressed exciton behaves as if the aggregate FC factor in Eq. (17) is unity. This follows from a cancellation of the actual FC factor arising from the $\widetilde{v}$ sums in Eq. (13b) with the FC factor contributed by $I^{0-0}$ [see Eq. (18)] in the denominator of Eq. (13b). Since, for the 0-0 line the excitonic coupling strength, $J_{0}$, enters only implicitly 
through the FC factor, we conclude that $g_{\text {lum }}^{0-0}$ is insensitive to both $\lambda$ and $J_{0}$ as is apparent from Eqs. (20) and (21).

Figure 3(b) shows the behavior of $g_{\text {lum }}^{0-0}$ as a function of $N$ as calculated using both Eqs. (20) and (21). When $N \phi$ $\ll 1, g_{\text {lum }}^{0-0}$ behaves as $2 k d / \phi-k d \phi N^{2} / 4$. When the size increases to $N \approx \operatorname{int}(\pi /|\phi|)$ (while maintaining $|\phi| \ll 1$ ), the dissymmetry passes through zero and changes sign. $g_{\text {lum }}^{0-0}$ from Eq. (20) contains an apparent divergence whenever $N \phi$ is exactly $2 \pi l(l=0,1,2, \ldots)$ due to the vanishing of the $0-0$ emission intensity at these points. However, the more general form of the dissymmetry in Eq. (21) contains a correction of order $\mathrm{O}\left[(N-1)^{2}(k d)^{2}\right]$ in the denominator which prevents divergences, causing $g_{\text {lum }}^{0-0}$ to instead nearly vanish when $N \phi=2 \pi l$.

The separation of the electronic and vibrational degrees of freedom unfortunately does not extend to the replica intensities. Moreover, unlike $g_{\text {lum }}^{0-0}, g_{\text {lum }}^{0-1}$ is very sensitive to the excitonic coupling strength. In what follows, we investigate the limiting forms of the $0-0$ and $0-1$ line strengths and dissymmeties in both the weak and strong exciton coupling limits. We continue to employ pbc's with even $N$.

\section{A. Weak coupling limit}

When the free exciton bandwidth, $4 J_{0}$ (as measured without electron-phonon coupling), is much smaller than the vibrational frequency, $\omega_{0}$, the excitonic coupling term in $H$ of Eq. (1) can be treated as a perturbation. In this limit the excited states form bands of vibronic excitons with band centers at energies $\omega_{0-0}+D+\widetilde{v} \omega_{0}(\widetilde{v}=0,1,2, \ldots)$ above the vibrationless ground state, and with narrow bandwidths approximately given by $4 J_{0} f_{0 \tilde{v}}^{2}$. Emission proceeds from the lowest exciton in the $\widetilde{v}=0$ band as long as $k T$ remains much smaller than the $\widetilde{v}=0$ bandwidth, $4 J_{0} e^{-\lambda^{2}}$. Thus, in this section we treat the limit, $k T e^{\lambda^{2}} \ll 4 J_{0} \ll \omega_{0}$.

In the weak coupling limit and under pbc's the singleparticle coefficients from Eq. (15) are given by $c_{n, \tilde{v}}^{(e m)}$ $\approx(-1)^{n} \delta_{\tilde{v}, 0}$, to zeroth order in $J_{0} / \omega_{0}$. The emitting exciton is vibrationally relaxed with the vibronic excitation residing almost entirely in the $\widetilde{v}=0$ vibrational level of the $1^{1} B_{u}$ electronic excited state. From Eq. (17) the FC factor is then simply

$$
F=\exp \left(-\lambda^{2}\right), \quad 4 J_{0} \ll \omega_{0} .
$$

Substituting the FC factor in Eq. (22) into Eqs. (18) gives directly the 0-0 line strengths. The $0-0$ dissymmetry factor remains that given by Eq. (20) or (21), which, as stated earlier, persists for any coupling strength $J_{0}$.

The independence of $g_{\text {lum }}^{0-0}$ on the intermolecular coupling is at first somewhat unsettling since one might expect that as $J_{0}$ vanishes so too must $g_{\text {lum }}^{0-0}$, since the isolated molecules are taken to be achiral. However, we have assumed from the start of this subsection that the band width remains larger than $k T$ so that emission emanates solely from the lowest energy exciton, which, for $J_{0}>0$, has wave vector $k$ $=\pi$. If this assumption is relaxed (by either raising the temperature or reducing $J_{0}$ ), thermally activated emission arises from higher energy excitons with $k \neq \pi$. The resulting inter- ference will cause the dissymmetry factor to vanish, as expected.

The 0-1 line strength and dissymmetry factor depend on one-and two-particle coefficients. In the weak coupling regime, the (small) two-particle coefficients appear to first order in $J_{0} / \omega_{0}$. Evaluating the first-order two-particle coefficients and inserting into Eqs. (14a) and (14b) gives the 0-1 line strength and dissymmetry factors:

$$
I^{0-1}=\lambda^{2} e^{-\lambda^{2}}+O\left[J_{0} / \omega\right]
$$

and

$$
\begin{aligned}
g_{\text {lum }}^{0-1}= & \frac{4 k d e^{-\lambda^{2}}\left(1-e^{-\lambda^{2}}\right)}{N I^{0-1}} \sum_{n>n^{\prime}}\left(J_{n n^{\prime}} / \omega_{0}\right)\left(n-n^{\prime}\right) \\
& \times \sin \left[\phi\left(n-n^{\prime}\right)\right]+O\left[\left(J_{0} / \omega_{0}\right)^{2}\right] .
\end{aligned}
$$

To lowest order in $J_{0} / \omega_{0}$ the intensity of the first vibronic replica is entirely independent of $N$ and is equal to the isolated molecule value. In fact, to zeroth order, it can be further shown that the total intensity of all replicas in the weak coupling limit attain their single-molecule values:

$$
I^{0-v_{t}}=\lambda^{2 v_{t}} e^{-\lambda^{2} / v_{t} !, \quad v_{t} \geqslant 1 .}
$$

Comparison with Eq. (18) shows that when $N \phi>1$, the replica intensities greatly exceed the origin $(0-0)$ emission.

Finally, performing the sums in Eq. (23b) gives the firstorder dissymmetry factor for the first vibronic replica,

$$
\begin{aligned}
g_{\text {lum }}^{0-1}= & \frac{4 k d\left(1-e^{-\lambda^{2}}\right)(N-1)}{N \lambda^{2}} \\
& \times\left(J_{0} / \omega_{0}\right)\{\sin \phi+\sin [(N-1) \phi]\}, \quad N>2 .
\end{aligned}
$$

[For a dimer, with $N=2, g_{\text {lum }}^{0-1}$ from Eq. (25) must be divided by 2.] In the weak coupling limit $g_{\text {lum }}^{0-1}$ initially increases linearly with $J_{0} / \omega_{0}$, thereby providing a measure of the exciton bandwidth. When $J_{0} / \omega_{0}$ increases beyond the weak coupling regime the dependence of $g_{\text {lum }}^{0-1}$ on $J_{0} / \omega_{0}$ grows weaker, eventually converging to the bandwidth independent 0-0 dissymmetry, $g_{\text {lum }}^{0-0}$, as the strong coupling regime is approached (see below).

\section{B. Strong coupling limit}

In the strong coupling limit, the nearest neighbor excitonic coupling is much greater than the vibrational energy, $J_{0} \gg \omega_{0}$. In this limit the exciton-phonon coupling term, which can be rewritten from Eq. (1) as

$$
H^{\prime}=\frac{\omega_{0} \lambda}{\sqrt{N}} \sum_{k} \sum_{q(\neq 0)}|k+q\rangle\langle k| b_{q}+\text { h.c., }
$$

becomes the perturbation. In Eq. (26), $|k\rangle=N^{-1 / 2} \sum_{n} e^{i k n}|n\rangle$ is a pure exciton with wave vector $k(k=0, \pm 2 \pi / N, \ldots, \pi$ assuming $N$ even) and $b_{q}$ is the destruction operator for a phonon with wave vector $q(q=0, \pm 2 \pi / N, \ldots, \pi)$. Note that the summation over $q$ does not include the $q=0$ totally symmetric phonon which can be treated exactly within the unperturbed part of the Hamiltonian. 
The zeroth-order multiparticle states are products of pure exciton and pure phonon parts. Accordingly, the emitting state takes the form

$$
\left|\Psi^{(e m)}\right\rangle \approx|k=\pi\rangle \otimes|v a c\rangle,
$$

to zeroth order, where $|v a c\rangle$ is the vacuum phonon state, written as $|v a c\rangle=\left|\tilde{\tilde{0}}_{0} \ldots 0_{q} \ldots 0_{q=\pi}\right\rangle$, indicating that all $N$ phonon modes have no quanta. The totally symmetric $q=0$ phonon is uncoupled from the electronic variables, and admits to an exact solution which dictates a shifted nuclear potential by $\lambda / \sqrt{N}$ relative to the harmonic well minimum in the electronic ground state. The double tilde refers to this slightly shifted nuclear potential. The shift can be neglected for sufficiently large aggregates.

Corrected to first order, the emitting exciton becomes

$$
\begin{aligned}
\left|\Psi^{(e m)}\right\rangle \approx \mid k & =\pi\rangle \otimes \mid \text { vac }\rangle \\
+ & \frac{\omega_{0} \lambda}{\sqrt{N}} \sum_{q \neq 0} \frac{|\pi-q\rangle \otimes b_{q}^{\dagger}|v a c\rangle}{E_{\pi}-\left(E_{\pi-q}+\omega_{0}\right)},
\end{aligned}
$$

where the zeroth-order exciton energy is $E_{k}=\omega_{0-0}+D$ $+2 J_{0} \cos k$. Instead of rewriting the emitting wave function (28) in the multi-particle basis set and then utilizing Eqs. (13a) - (14b) to evaluate the $0-0$ and $0-1$ emission characteristics, we simply insert the wave function (28) directly into the general expressions for the line strength and magnetic dipole moments, Eqs. (4) and (11), respectively. For the 0-0 line strength, we recover Eqs. (18), but with the FC factor given by

$$
F=\exp \left(-\lambda^{2} / N\right), \quad 4 J_{0} \gg \omega_{0} .
$$

which approaches unity as $N$ increases. We also exactly recover the 0-0 dissymmetry as given by Eq. (20). Hence, in the strong coupling limit, the $0-0$ emission refects free exciton behavior, in which the electronic excitation is uncoupled from molecular vibrations.

Proceeding with the 0-1 emission, we obtain the 0-1 line strength,

$$
\begin{aligned}
I^{0-1}= & \left(\lambda^{2} e^{-\lambda^{2} / N} / N^{2} \mu_{0}^{2}\right) \\
& \times \sum_{\beta} \sum_{q=0, \pm 2 \pi / N, \ldots} \frac{\left|\Sigma_{n} \mu_{n, \beta} e^{i(\pi-q) n}\right|^{2}}{\left[4\left(J_{0} / \omega_{0}\right) \sin ^{2}(q / 2)+1\right]^{2}} .
\end{aligned}
$$

When $J_{0} / \omega_{0} \gg\left[4 \sin ^{2}(\pi / N)\right]^{-1}$, the $q=0$ term in the sum dominates and the total 0-1 line strength reduces to

$$
I^{0-1}=\left(\lambda^{2} e^{-\lambda^{2} / N} / N^{2}\right) \frac{\sin ^{2}(N \phi / 2)}{\cos ^{2}(\phi / 2)},
$$

which is $N$ times smaller than the $0-0$ intensity in Eq. (18). This behavior contrasts that in the weak coupling regime, where the $0-1$ intensity is much larger and essentially size independent [see Eq. (24)]. Furthermore, in the strong coupling regime the origin and all the replicas are zero whenever $N \phi=2 \pi l$ (with $l=1,2, \ldots$ ), in contrast to the weak coupling regime where only the 0-0 line strength vanishes when $N \phi$ $=2 \pi l$. In fact, all of the replica line strengths are related to the $0-0$ line strength through

$$
\begin{aligned}
I^{0-v_{t}} & =\lambda^{2 v_{t}} I^{0-0} / v_{t} ! N^{v_{t}}, \\
v_{t} & =0,1, \ldots, \quad J_{0} / \omega_{0} \gg\left[4 \sin ^{2}(\pi / N)\right]^{-1} .
\end{aligned}
$$

Equation (32) shows that as the aggregate size increases the emission spectrum becomes dominated by the $0-0$ peak, in sharp contrast to what is observed in the weak coupling limit.

Finally, in the strong coupling limit the $0-1$ dissymmetry factor becomes

$$
g_{\text {lum }}^{0-1}=\frac{2 k d \lambda^{2} e^{-\lambda^{2} / N}}{N^{2} I^{0-1}} \sum_{q} \frac{\sum_{n>n^{\prime}}(-1)^{n-n^{\prime}}\left(n^{\prime}-n\right) \sin \left[\phi\left(n-n^{\prime}\right)\right] \cos \left[\left(n-n^{\prime}\right) q\right]}{\left[4\left(J_{0} / \omega_{0}\right) \sin ^{2}(q / 2)+1\right]^{2}} .
$$

Again, when $J_{0} / \omega_{0} \gg\left[4 \sin ^{2}(\pi / N)\right]^{-1}$, the largest contribution comes from the $q=0$ term which, after evaluating the summations over molecular sites, gives

$$
\begin{aligned}
g_{\text {lum }}^{0-1}=k d\{N \cot (N \phi / 2)+\tan (\phi / 2)\}, & \\
& J_{0} / \omega_{0} \gg|\lambda| N^{3 / 2} / 4 \pi^{2} .
\end{aligned}
$$

$g_{l u m}^{0-1}$ is identical to $g_{\text {lum }}^{0-0}$ in Eq. (20). In fact, all of the replica dissymmetries reduce to the $0-0$ value in the strong coupling limit,

$$
g_{\text {lum }}^{0-v_{t}}=g_{\text {lum }}^{0-0}, \quad J_{0} / \omega_{0} \gg\left[4 \sin ^{2}(\pi / N)\right]^{-1}, \quad v_{t}=0,1, \ldots .
$$

Therefore, in progressing from the weak to strong coupling regimes, the 0-0 dissymmetry factor remains constant, while the replica dissymmetries initially increase linearly with exciton bandwidth, eventually converging to the $0-0$ dissymmetry factor.

\section{Weak and strong field comparison}

The striking behavioral differences between $g_{\text {lum }}^{0-0}$ and $g_{\text {lum }}^{0-1}$ may be compared to studies of the linear polarization of light emission from aggregates, where the higher vibronic transitions are also predicted to have a polarization different from that of the $0-0$ transition. ${ }^{34,39,40}$ One way to appreciate the differences in the circular polarization dissymmetry as a function of exciton bandwidth is to rewrite the emitting exciton in the exciton-phonon basis set up to terms containing zero and one phonon. We obtain

$$
\begin{aligned}
\left|\Psi^{(e m)}\right\rangle \approx & e^{-\lambda^{2}}|k=\pi\rangle \otimes|v a c\rangle_{0} \\
& +\frac{\lambda^{2} e^{-\lambda^{2}}}{\sqrt{N}} \sum_{k}|k\rangle \otimes b_{\pi-k}^{\dagger}|v a c\rangle_{0},
\end{aligned}
$$

in the weak coupling limit, and 


$$
\begin{aligned}
\left|\Psi^{(e m)}\right\rangle \approx & e^{-\lambda^{2} / N}|k=\pi\rangle \otimes|v a c\rangle_{0} \\
& +\frac{\lambda^{2} e^{-\lambda^{2} / N}}{N}|k=\pi\rangle \otimes b_{q=0}^{\dagger}|v a c\rangle_{0}
\end{aligned}
$$

in the strong coupling limit, where $|v a c\rangle_{0}$ $\equiv\left|0_{0} \cdots 0_{q} \cdots 0_{q=\pi}\right\rangle$, i.e., a differing from $|v a c\rangle$ defined after Eq. (27) in that the totally symmetric phonon is not shifted.

In both limits, the $0-0$ emission properties arise entirely from the leading term in the wave function which differs in the two cases by the FC factor. Since $g_{\text {lum }}^{0-0}$ is independent of the FC factor, $g_{\text {lum }}^{0-0}$ is identical in the two limits. The $0-1$ dissymmetry arises from the second terms in both expressions. In the weak coupling limit, the sum over $k$ indicates that the dissymmetry consists of many pathways which destructively interfere. The interference is absent in the strong coupling limit, enabling $g_{\text {lum }}^{0-1}$ to be nonzero and, in fact, equal to $g_{\text {lum }}^{0-0}$.

The $g_{\text {lum }}^{0-0}-g_{\text {lum }}^{0-1}$ differences can also be interpreted in terms of "dynamic localization." The assumption that the emissive transitions are "vertical" implies that the 0-0 transition originates predominantly from instants during the excited state zeropoint vibrational motion where the nuclear geometry resembles that of the ground state with no phonons. In this geometry, all the chromophoric units are equal and the resonance between the chromophores causes the excitation to be delocalized over the entire aggregate. Under these conditions the luminescence is circularly polarized.

In the case of weak coupling, it is possible for the excitation to contract on almost a single chromophore during a particular phase of the zero point vibrational motion. In the associated geometry, the molecule carrying the excitation is strongly distorted with respect to its groundstate equilibrium geometry. The vertical transition back to the ground state ends mainly in the higher vibrational levels of the latter state. Furthermore, in the distorted geometry, the coupling between the transition dipole moments of the chromophoric molecules is weakened due to the fact that the distorted chromophore is out of resonance with the other chromophores. Thus, in the distorted geometries, the emission originates mainly from a single chromophore and has therefore almost no net circular polarization. When the excitonic coupling is strong enough localization is prevented leading to a much larger dissymmetry for the $0-1$ transition.

Thus, the different degree of circular polarization for the $0-0$ and $0-1$ vibronic transitions indicates that "size" of the excitation fluctuates during the excited state zeropoint vibrational motion and that the excitation can localize momentarily on a single chromophoric unit.

\section{NUMERICAL RESULTS}

All of the limiting behaviors for the weak and strong coupling regimes outlined in the last section, as well as the important intermediate regime, can be demonstrated through the numerical analysis of the Hamiltonian in Eq. (1) and subsequent use of Eqs. (4) and (8) to obtain the emission spectrum as well as dissymmetry factors for all vibronic peaks. We utilize Eq. (8) instead of (11) for the dissymmetry

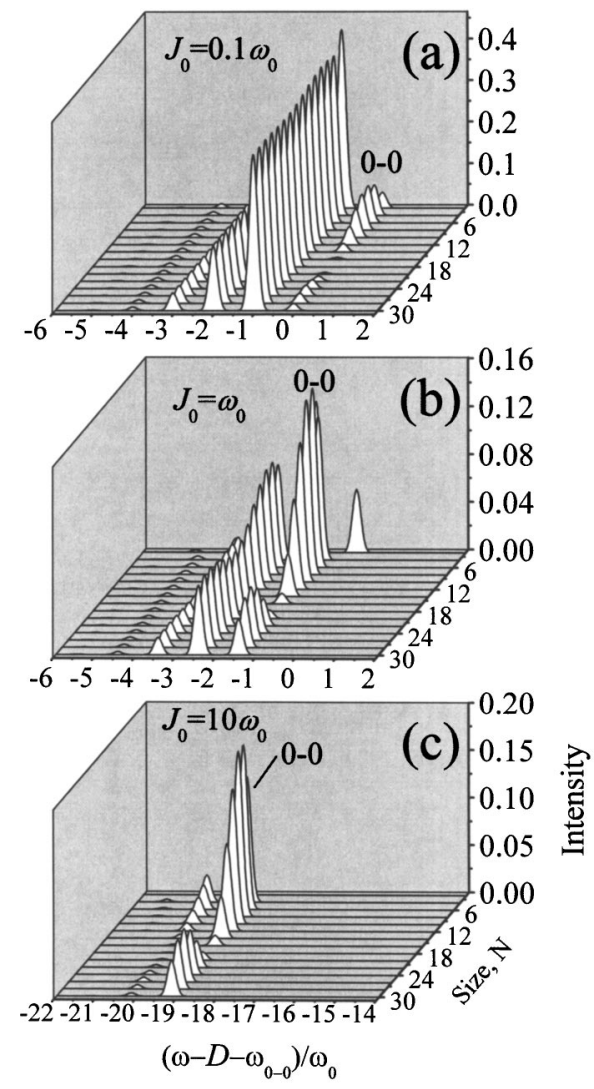

FIG. 4. Emission spectra as a function of $N$ obtained by numerical analysis of $H$ and subsequent use of Eqs. (3) and (4) for (a) weak coupling $\left(J_{0} / \omega_{0}\right.$ $=0.1) ;\left(\right.$ b) intermediate coupling $\left(J_{0} / \omega_{0}=1\right)$; and (c) strong coupling case $\left(J_{0} / \omega_{0}=10\right)$. In all cases the Huang-Rhys factor, $\lambda^{2}$, was set to unity and $\omega_{0}=\left(\omega_{0-0}+D\right) / 20$. Note that the dimer $(N=2)$ emission in (b) is blueshifted because the exciton bandwidth is reduced by about a factor of 2 compared to larger aggregates. In (c) the dimer spectrum is completely shifted out of the spectral window and is not observed.

factors to avoid unphysical divergences at the intensity minima. The emission transition frequency, $\omega_{e m}$, as well as the one-, two-, and three-particle coefficients of the emitting state were obtained by numerically diagonalizing the Hamiltonian (1) using standard IMSL packages. In what follows we take $\phi=\pi / 9$ and $k d=0.01$ and utilize a maximum of five vibrational quanta which is enough to ensure convergence of all observables. In addition, the vibrational frequency, $\omega_{0}$, is set to $\left(\omega_{0-0}+D\right) / 20$ and the Huang-Rhys factor, $\lambda^{2}$, is set to unity, as is representative of many conjugated organic chromophores.

We begin with the unpolarized emission spectrum, $S(\omega)$, calculated from Eq. (4). Figure 4 shows $S(\omega)$ as a function of aggregate size, $N$, for three values of the nearest neighbor coupling; $J_{0}=0.1 \omega_{0}, \omega_{0}$, and $10 \omega_{0}$, corresponding to Figs. 4(a)-4(c), respectively, and representative of the weak, intermediate, and strong coupling regimes. In Fig. 4(a), the emission spectrum is essentially that of a single molecule at all sizes with the exception of the 0-0 line which undergoes a damped oscillation according to Eq. (18). At $N$ $=18$ the aggregate has undergone a full twist and the $0-0$ intensity vanishes. When $J_{0}$ increases tenfold, the intermediate coupling regime is reached. Figure 4(b) shows that $0-0$ peak continues to oscillate and diminish with roughly the 

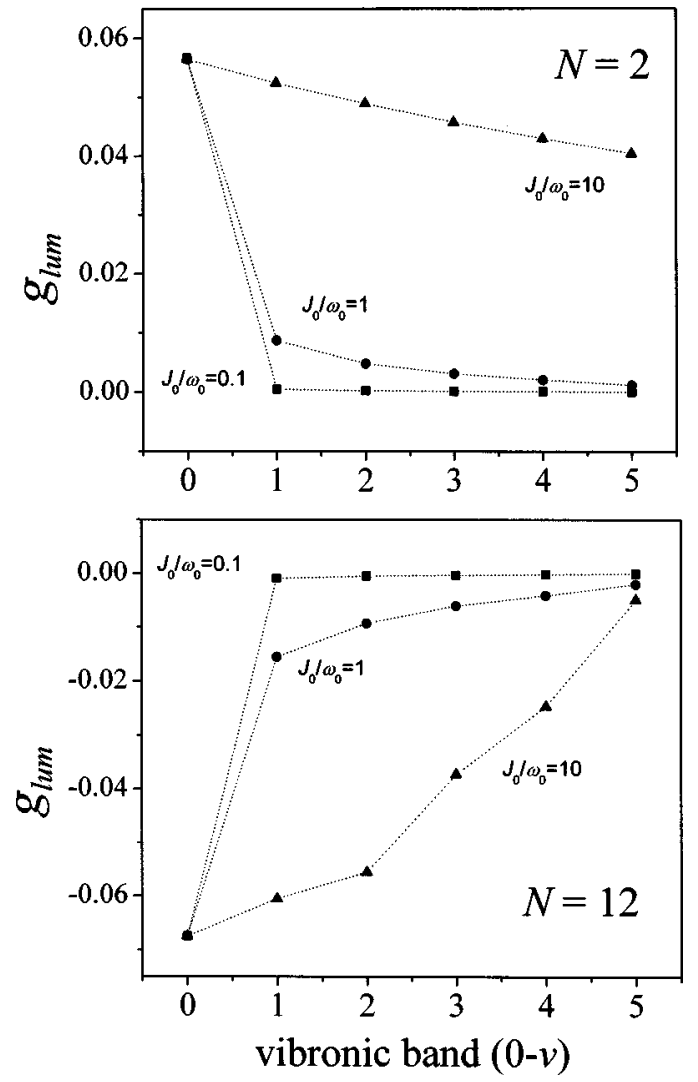

FIG. 5. Degree of circular polarization in emission for various vibronic transitions as obtained from numerical analysis and use of Eq. (8) of aggregates with $N=2$ and $N=12$ chromophores. Results for strong, intermediate, and weak coupling are shown. The dotted line only serves as a guide to the eye. In all cases $\lambda^{2}=1, \phi=\pi / 9$, and $k d=0.01$.

same peak magnitude as in Fig. 4(a), due to a small increase in the generalized FC factor in Eq. (17). The main difference from the weak coupling regime is the strong attenuation of the replica peaks by about an order of magnitude. The reduction is mainly a result of one- and two-exciton destructive interference as embodied in the expression (14a). Such a mechanism is unimportant in the weak coupling regime where the two-particle coefficients are negligible. In the strong coupling regime [see Fig. 4(c)] the entire spectrum oscillates and dampens with $N$; however, the $0-v_{t}$ replica remains of order $\mathrm{O}\left[N^{-} v_{t}\right]$ as large as the 0-0 peak [see Eq. (32)], the latter eventually dominating all replicas with increasing $N$. In contrast to the weak and intermediate regimes, the entire spectrum vanishes when condition (19) applies (at $N=18$ ) as required by Eq. (32).

The emission dissymmetry factors, $g_{\text {lum }}^{0-v_{t}}$, for the $0-0$ peak as well as four replicas are shown in Fig. 5 for $N=2$ and $N=12$ aggregates. All three coupling regimes are depicted. For $N=2$ the circular polarization dissymmetry is positive for all peaks while for $N=12$ it is negative. (The reverse holds for aggregates with pitch angle $-\phi$.) $g_{\text {lum }}^{0-0}$ is entirely independent of $J_{0}$ as predicted by Eqs. (20) and (21). In the weak coupling regime $g_{\text {lum }}^{0-0}$ is dominant. As $J_{0}$ increases the replica $g_{\text {lum }}$ 's approach $g_{\text {lum }}^{0-0}$, consistent with Eq. (35). Although not shown, by $J_{0}=1000 \omega_{0}$ there is essentially no change in $g_{\text {lum }}^{0-v_{t}}$ with $v_{t}$.

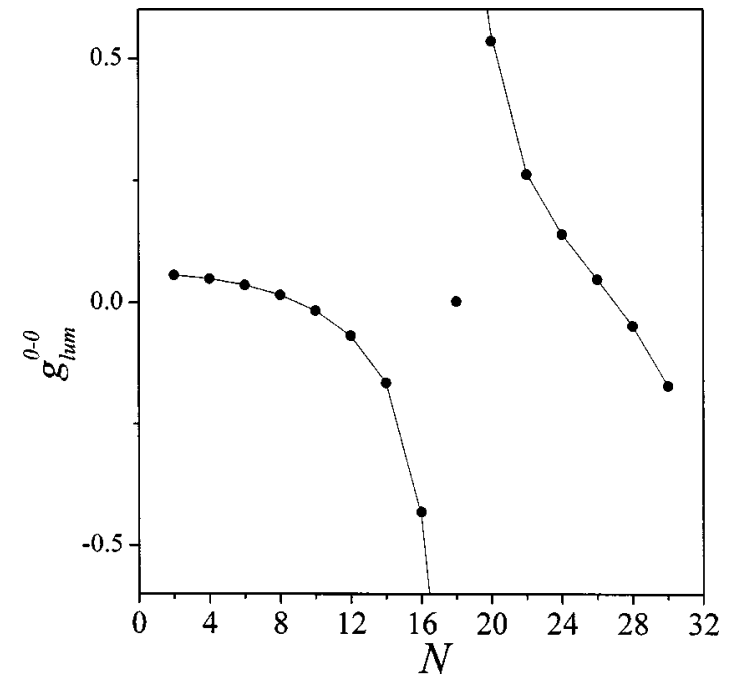

FIG. 6. Degree of circular polarization in the 0-0 emission $\left(g_{\text {lum }}^{0-0}\right)$ as a function of aggregate size as calculated numerically using Eq. (8) (solid circles) and analytically using Eq. (20) (solid line). In all cases $\lambda^{2}=1, \phi$ $=\pi / 9$, and $k d=0.01$. The numerical results do not diverge at $N=18$ due to extended size effects (see text).

To illustrate the size dependence further, Figs. 6 and 7 show $g_{\text {lum }}^{0-0}$ and $g_{\text {lum }}^{0-1}$, respectively, as a function of $N$. Again, for the $0-1$ band, the three coupling regimes are illustrated. The calculations show that $g_{\text {lum }}^{0-0}$ changes sign at $N=10, N$ $=18$, and $N=28$ in good agreement with what is predicted from Eq. (20). The sign reversal at $N=10$ and $N=28$ is related to the fact that a stack with this number of chromophores has just completed a half turn $\left(\phi=20^{\circ}\right)$. At $N$ $=18$ a full turn is completed, causing the $0-0$ transition dipole to become vanishingly small, driving the $0-0$ intensity to zero, and creating an apparent divergence in $g_{\text {lum }}^{0-0}$ as evaluated using Eq. (20). However, the numerical evaluation of $g_{\text {lum }}^{0-0}$ using Eq. (8) has no such divergence at $N=18$. Nevertheless, $\left|g_{\text {lum }}^{0-0}\right|$ for aggregates with $N=16$ and $N=20$ becomes very large using either Eq. (8) or (20). Normally, for organic chromophores with dipole allowed transitions, the electric dipole moments are much larger in magnitude than the magnetic dipole moments, resulting in small $g_{\text {lum }}$ values [see Eq. (11)]. However, as the net transition dipole moment is strongly reduced for the aggregates with $N=16$ and $N$ $=20$, a large $\left|g_{\text {lum }}^{0-0}\right|$ results.

For the case of strong coupling, the $N$ dependence of $g_{l u m}^{0-1}$ closely follows that of $g_{\text {lum }}^{0-0}$. This is further illustrated in Fig. 8, which shows that $\left|g_{\text {lum }}^{0-1} / g_{\text {lum }}^{0-0}\right|$ is slightly less than unity for $J_{0} / \omega_{0}=10$. For the case of weak coupling $\left|g_{\text {lum }}^{0-1} / g_{\text {lum }}^{0-0}\right|$ is small, consistent with the view that the $(0-1)$ vibronic emission originates mainly from nuclear geometries sampled during the excited zero point vibrational motion in which the excitation is mainly localized on a single chromophore. In the intermediate case $\left|g_{\text {lum }}^{0-1} / g_{\text {lum }}^{0-0}\right|$ varies considerably with $N$ but is always smaller than one. Especially near $N=8,\left|g_{\text {lum }}^{0-1} / g_{\text {lum }}^{0-0}\right|$ reaches high values, due to the sign change between $N=8$ and $N=10$ of the magnetic dipole moment (and hence $g_{l u m}^{0-0}$ ). For a completely delocalized excitation with $N=8$, the net magnetic dipole moment is relatively small because contributions from individual chro- 

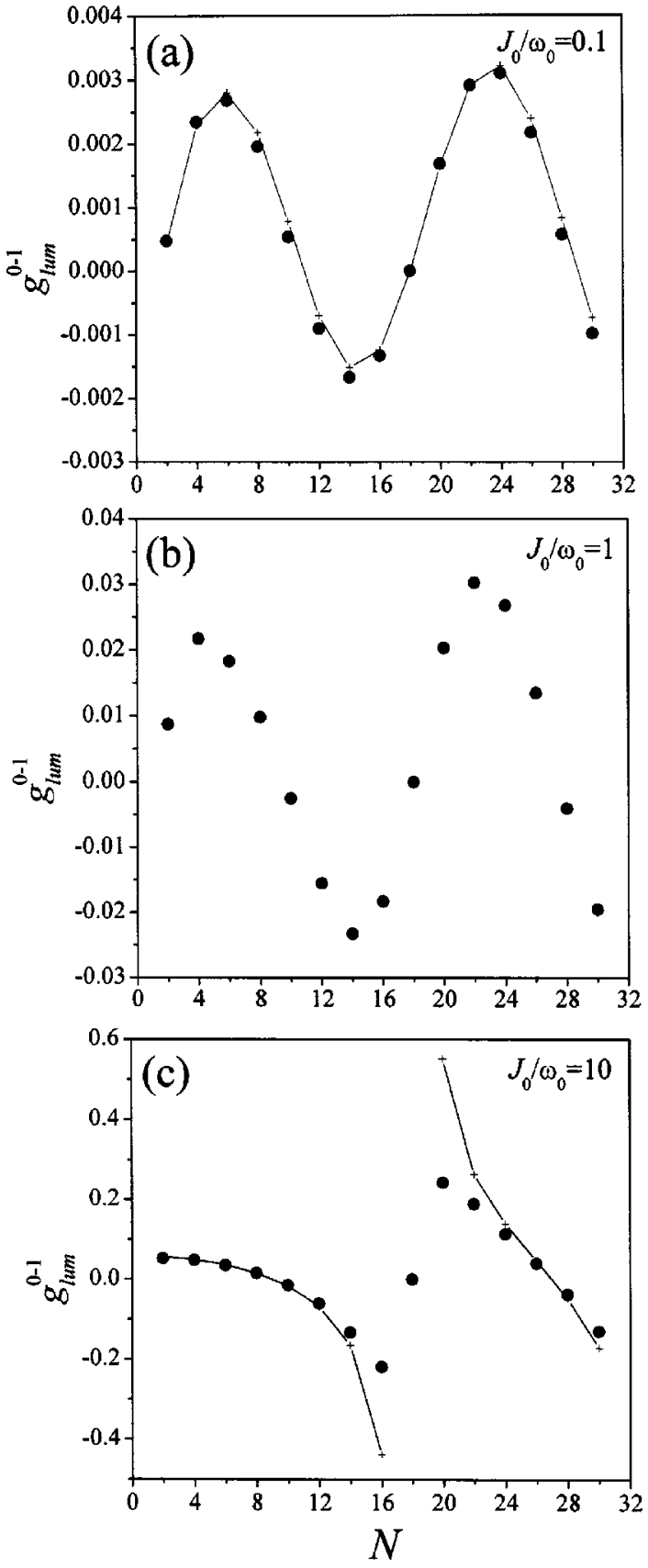

FIG. 7. Degree of circular polarization in the $0-1$ emission $\left(g_{l u m}^{0-1}\right)$ as a function of aggregate size as calculated numerically (solid circles) for the weak (a), intermediate (b), and strong (c) excitonic coupling regimes. In (a) and (c), the crosses with connecting solid lines represent the analytical results in Eqs. (25) and (34), respectively. In all cases $\lambda^{2}=1, \phi=\pi / 9$, and $k d=0.01$.

mophores cancel each other out. In the distorted geometries which mainly give rise to the higher vibronic transitions, the excitation is more contracted and the magnitude of the magnetic dipole moment is less due to cancellation effects. Finally, Fig. 9 shows how $g_{l u m}^{0-1} / g_{\text {lum }}^{0-0}$ changes with $J_{0} / \omega_{0}$ for several aggregate sizes. The initial increase is linear as predicted by Eq. (25), and the saturation to unity in the strong coupling limit is consistent with Eq. (34).

The preceding analysis shows that dissymmetry measurements can be used to measure the exciton coherence size as well as bandwidth in chiral aggregates. As shown by Eq.

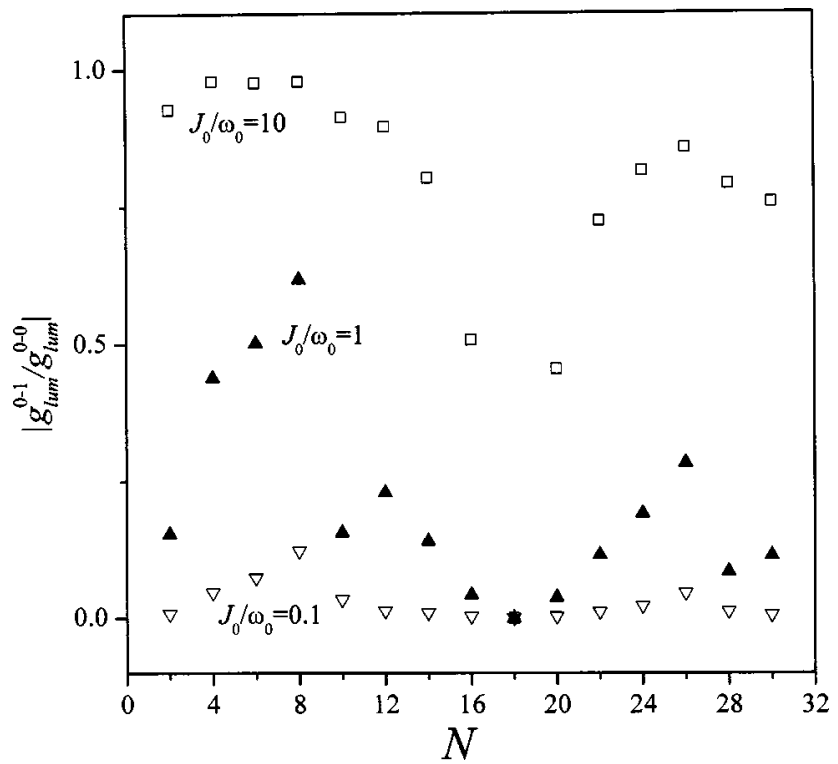

FIG. 8. The ratio, $\left|g_{\text {lum }}^{0-1} / g_{\text {lum }}^{0-0}\right|$, calculated numerically, as a function of aggregate size $N$ for $J_{0} / \omega_{0}=10(\square), J_{0} / \omega_{0}=1(\mathbf{\Lambda})$, and $J_{0} / \omega_{0}=0.1(\nabla)$. In all cases $\lambda^{2}=1, \phi=\pi / 9$, and $k d=0.01$.

(20), $g_{\text {lum }}^{0-0}$ is completely independent of $J_{0}$ and $\lambda^{2}$ but is sensitive to aggregate geometry, i.e., $N, \phi$, and $d . g_{\text {lum }}^{0-0}$ therefore provides an estimate of the exciton coherence size, $N$, if $\phi$ and $d$ are already known. Given the aggregate structural parameters the exciton bandwidth can then be deduced from the ratio $g_{\text {lum }}^{0-1} / g_{\text {lum }}^{0-0}$, which is a sensitive function of $J_{0}$, as shown in Fig. 9.

\section{COMPARISON TO EXPERIMENT AND CONCLUDING REMARKS}

In this section we will briefly compare the theoretical predictions made above to published experimental data. Dedicated experimental studies are in progress and will be published elsewhere. From fluorescence studies on $\pi$-conjugated oligomers of, e.g., thiophene ${ }^{41}$ and

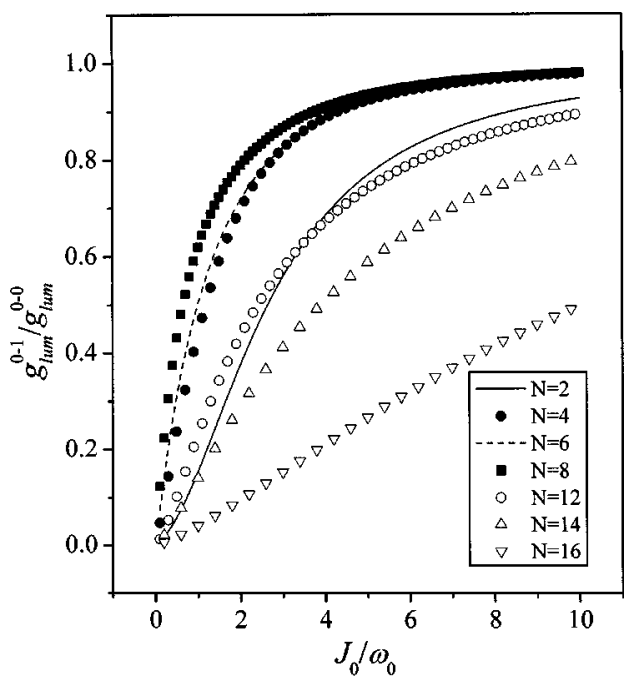

FIG. 9. $g_{\text {lum }}^{0-1} / g_{\text {lum }}^{0-0}$ calculated numerically, as a function of $J_{0} / \omega_{0}$ for several aggregate sizes. $\lambda^{2}=1, \phi=\pi / 9$, and $k d=0.01$. 
phenylenevinylene, ${ }^{42}$ it is well know that typically the excitation on an isolated oligomer couples to an intramolecular vibration (or set of vibrations) with frequency of $\sim 1400$ $\mathrm{cm}^{-1}$. Upon aggregation, photophysical properties of the aggregates change drastically, which is in many cases interpreted in terms of formation of a band of exciton states with a width on the order of $10^{3} \mathrm{~cm}^{-1} .{ }^{43}$ These numbers indicate that the intermediate coupling regime is expected to apply for aggregates of $\pi$-conjugated oligomers, and a reduction of the absolute value of $g_{\text {lum }}$ with decreasing photon energy is predicted. Experiments on a model compound containing two terthiophene chromophoric units indeed showed strong reduction of $\left|g_{\text {lum }}\right|$ with increasing wavelength of fluorescence detection. ${ }^{44}$ This indicates that the intermediate coupling case applies to this chromophore system. A decrease of $\left|g_{\text {lum }}\right|$ with decreasing photon energy has been observed for nano-aggregates ${ }^{45}$ and for a solid film ${ }^{46}$ of a polythiophene derivative. Similar effects have been seen for nanoaggregates of a chiral poly(phenylene)vinylene. For the latter material the effect has also been seen for both electro- and photo-luminescence ${ }^{47}$ from solid films. Thus for these materials also the intermediate coupling regime seems to apply. Analysis of data for polymers is considerably more difficult because energy transfer processes may result in spectral diffusion of the emission band. Therefore, fluorescence may originate from trap sites which are not necessarily representative of the bulk behavior of the material. In conclusion, experimental data confirm the theoretical prediction of a reduction of $\left|g_{\text {lum }}\right|$ for higher vibronic transitions compared to $g_{\text {lum }}$ for the 0-0 transition; more detailed studies are underway.

\section{ACKNOWLEDGMENTS}

F.C.S. is supported by the National Science Foundation Grant No. DMR \#0305173. The research of S.C.J.M. has been made possible by a fellowship from the Royal Dutch Academy of Sciences.

${ }^{1}$ A. Kohler, J. S. Wilson, and R. H. Friend, Adv. Mat. 14, 701 (2002).

${ }^{2}$ U. Scherf and E. J. W. List, Adv. Mat. 14, 477 (2002).

${ }^{3}$ A. J. Heeger, J. Phys. Chem. B 105, 8475 (2001).

${ }^{4}$ B. J. Schwartz, Annu. Rev. Phys. Chem. 54, 141 (2003).

${ }^{5}$ L. Pu, Acta Polym. 48, 116 (1997).

${ }^{6}$ J. P. Riehl and F. S. Richardson, Chem. Rev. 86, 1 (1986).

${ }^{7}$ H. P. J. M. Dekkers, in Circular Diochroism, edited by K. Nakanishi, N. Berova, and R. W. Woody (Wiley-VCH, New York, 2001).

${ }^{8}$ B. M. W. Langeveld-Voss, R. A. J. Janssen, and E. W. Meijer, J. Mol. Struct. 521, 285 (2000).

${ }^{9}$ Y. Geng, A. Trajkovska, D. Katsis, J. J. Ou, S. W. Culligan, and S. H. Chen, J. Am. Chem. Soc. 124, 8337 (2002).
${ }^{10}$ A. Rajca, H. Wang, V. Pawitranon, T. J. Brett, and J. L. Stezowski, Chem. Commun. (Cambridge) 12, 1060 (2001).

${ }^{11}$ O. Henze, M. Fransen, P. Jonkheijm, E. W. Meijer, W. J. Feast, and A. P. H. J. Schenning, J. Polym. Sci. A 41, 1737 (2003).

${ }^{12}$ L. M. Herz, C. Daniel, C. Silva, F. Hoeben, A. P. H. J. Schenning, E. W. Meijer, R. H. Friend, and R. T. Phillips, Phys. Rev. B 68, 045203 (2003).

${ }^{13}$ D. Wang, T. J. Liu, C. J. Li, and W. T. Slaven, Polym. Bull. (Berlin) 39, 265 (1997).

${ }^{14}$ R. A. Garoff, E. A. Litzinger, R. E. Connor, I. Fishman, and B. A. Armitage, Langmuir 18, 6330 (2002).

${ }^{15}$ S. Lampoura, C. Spitz, S. Daehne, J. Knoester, and K. Duppen, J. Phys. Chem. B 106, 3103 (2002).

${ }^{16}$ H. Goto, N. Harada, J. Crassous, and F. Diederich, J. Chem. Soc., Perkin Trans. 2 8, 1719 (1998).

${ }^{17}$ A. E. Hansen and K. L. Bak, J. Phys. Chem. 104, 11362 (2000).

${ }^{18}$ M. R. Philpott, Adv. Chem. Phys. 23, 227 (1973).

${ }^{19}$ C. Didraga, J. A. Klugkist, and J. Knoester, J. Phys. Chem. B 106, 11474 (2002).

${ }^{20}$ I. Tinoco, Adv. Chem. Phys. 4, 113 (1962).

${ }^{21}$ K. Nakanishi, R. W. Woody, and N. Berova, Circular Dichroism (WileyVCH, New York, 2000)

${ }^{22}$ O. E. Weigang, Jr., J. Chem. Phys. 43, 71 (1965).

${ }^{23}$ R. L. Fulton and M. Gouterman, J. Chem. Phys. 35, 1059 (1961).

${ }^{24}$ A. Witkowski and W. Moffitt, J. Chem. Phys. 33, 872 (1960).

${ }^{25}$ N. Lu and S. Mukamel, J. Chem. Phys. 95, 1588 (1991).

${ }^{26}$ E. W. Knapp, P. O. J. Scherer, and S. F. Fischer, Chem. Phys. Lett. 111, 481 (1984).

${ }^{27}$ P. O. J. Scherer, Adv. Mat. 7, 451 (1995).

${ }^{28}$ F. C. Spano, J. Chem. Phys. 118, 981 (2003).

${ }^{29}$ M. Sterzel, M. Pilch, M. T. Pawlikowski, P. Skowronek, and J. Gawronski, Chem. Phys. Lett. 362, 243 (2002).

${ }^{30}$ M. Sterzel, M. Pilch, M. T. Pawlikowski, and J. Gawronski, J. Chem. Phys. 291, 251 (2003).

${ }^{31}$ M. Pawlikowski and M. Z. Zgierski, J. Chem. Phys. 76, 4789 (1982).

${ }^{32}$ M. Z. Zgierski and M. J. Pawlikowski, J. Chem. Phys. 79, 1616 (1983).

${ }^{33}$ M. R. Philpott, J. Chem. Phys. 55, 2039 (1971).

${ }^{34}$ F. C. Spano, J. Chem. Phys. 116, 5877 (2002).

${ }^{35}$ M. Hoffmann and Z. G. Soos, Phys. Rev. B 66, 024305 (2002).

${ }^{36}$ F. S. Richardson and J. P. Riehl, Chem. Rev. 77, 773 (1977).

${ }^{37}$ N. Harada and K. Nakanishi, Circular Dichroic Spectroscopy (University Science Books, Oxford, 1983).

${ }^{38}$ D. P. Craig and T. Thirunamachandran, Molecular Quantum Electrodynamics (Dover, New York, 1998).

${ }^{39}$ F. C. Spano, Chem. Phys. Lett. 331, 7 (2000).

${ }^{40}$ F. C. Spano, J. Chem. Phys. 114, 5376 (2001).

${ }^{41}$ R. S. Becker, J. Seixas de Melo, A. L. Macanita, and F. Elisei, J. Phys. Chem. 100, 18683 (1996).

${ }^{42}$ R. Mahrt, J. P. Yang, A. Greiner, H. Baessler, and D. D. C. Bradley, Macromol. Chem. Rapid Comm. 11, 415 (1990).

${ }^{43}$ M. Muccini, E. Lunedei, C. Taliani, D. Beljonne, J. Cornil, and J.-L. Bredas, J. Chem. Phys. 109, 10513 (1998).

${ }^{44}$ B. M. W. Langeveld-Voss, D. Beljonne, Z. Shuai, R. A. J. Janssen, S. C. J. Meskers, E. W. Meijer, and J. L. Bredas, Adv. Mat. 10, 1343 (1998).

${ }^{45}$ B. M. W. Langeveld-Voss, R. A. J. Janssen, M. P. T. Christiaans, S. C. J. Meskers, H. P. J. M. Dekkers, and E. W. Meijer, J. Am. Chem. Soc. 118, 4908 (1996).

${ }^{46}$ S. C. J. Meskers, E. Peeters, B. M. W. Langeveld-Voss, and R. A. J. Janssen, Adv. Mat. 12, 589 (2000).

${ }^{47}$ E. Peeters, M. P. T. Christiaans, R. A. J. Janssen, H. F. M. Schoo, H. P. J. M. Dekkers, and E. W. Meijer, J. Am. Chem. Soc. 119, 9909 (1997). 